\title{
Autoantibody Response to ZRF1 and KRR1 SEREX Antigens in Patients with Breast Tumors of Different Histological Types and Grades
}

\author{
Lada Dyachenko, ${ }^{1}$ Kristina Havrysh, ${ }^{2}$ Anita Lytovchenko, ${ }^{1}$ Irina Dosenko, ${ }^{3}$ \\ Stepan Antoniuk, ${ }^{4}$ Valeriy Filonenko, ${ }^{1}$ and Ramziya Kiyamova ${ }^{1,2}$ \\ ${ }^{1}$ Department of Cell Signaling, Institute of Molecular Biology and Genetics NASU, Kyiv, Ukraine \\ ${ }^{2}$ Institute of Fundamental Medicine and Biology, Kazan Federal University, Kazan, Russia \\ ${ }^{3}$ Breast Cancer Department, National Cancer Institute, Kyiv, Ukraine \\ ${ }^{4}$ Department of Oncological Pathology, Dnipropetrovsk Regional Center of Pathology, Dnipropetrovsk, Ukraine \\ Correspondence should be addressed to Ramziya Kiyamova; rgkiyamova@kpfu.ru
}

Received 20 June 2016; Revised 30 August 2016; Accepted 29 September 2016

Academic Editor: Szilárd Nemes

Copyright ( 2016 Lada Dyachenko et al. This is an open access article distributed under the Creative Commons Attribution License, which permits unrestricted use, distribution, and reproduction in any medium, provided the original work is properly cited.

\begin{abstract}
Purpose. To investigate a frequency of antibody response to SEREX-identified medullary breast carcinoma autoantigens ZRF1 and KRR1 in sera of breast cancer patients taking into account clinical and molecular characteristics of tumors for opening of new perspectives in creation of minimally invasive immunological tests for cancer diagnostics. Methods. Enzyme-linked immunosorbent assay and bioinformatics analysis. Results. Increased frequency of antibody response was found in sera of breast cancer patients to ZRF and KRR1 antigens. The antibody response to these antigens was higher in sera of patients with invasive ductal carcinoma than in sera of patients with other histological types of breast tumors. Moreover, more frequent antibody response to ZRF antigen was found in sera of patients with less aggressive tumors. The sequence analysis of ZRF1 antigen SEREX clones obtained from cDNA libraries of different tumors demonstrates that they encode different protein isoforms. Conclusion. Tumor-associated antigens KRR1 and ZRF1 and their cognate autoantibodies could be considered as potential molecular markers of breast cancer which need to be further investigated.
\end{abstract}

\section{Introduction}

Breast cancer is the most common female cancer in the world with high mortality rate because of high degree of malignancy and rapid proliferation. One of the main problems in breast cancer diagnostic is that traditional diagnostic methods, including mammography, ultrasound, magnetic resonance imaging, and clinical examination, are not sufficiently effective for tumor detection $[1,2]$. For example, mammography has a high rate of false-positive results and is characterized by insufficient sensitivity in some groups of women [3-5]. Such methods as ultrasound and magnetic resonance imaging are used as additional tools for tumors detection [3]. Moreover, some tumors can appear in periods between annual mammography screenings being difficult for detection by widely used screening technologies. So there is a need to search for new approaches including cancer biomarkers for breast tumors detection that could provide more effective, simple, and noninvasive diagnostic tools.

Recent advances in identification and characterization of tumor-associated antigens (TAAs) indicate that their correspondent autoantibodies are perspective cancer biomarkers, which have significant advantages compared to other biomarkers, in particular, the ability to be detected long before clinical manifestation of the disease and the stability and availability in blood serum, which open up opportunities to create minimally invasive cancer diagnostic immune assays $[5,6]$. According to the literature data, the detection rate of autoantibodies directed against single antigen in the sera of patients is low and ranges vary in average from 10 to $30 \%$. 
Therefore, the use of panel of TAAs, rather than individual TAAs, enhances the likelihood of cancer detection. It should be mentioned that a great number of antigenic panels created for breast cancer detection consist of well-known breast cancer TAAs, in particular p53, c-myc, Her2, Muc1, and survivin [7]. Most of them are known to be overexpressed in breast carcinoma tissues and can initiate autoimmune response [7-9]. Unfortunately, most of the known antigenic panels have insufficient sensitivity and specificity for breast cancer diagnostics, and no one of them was approved for use in clinic. The first commercial panel of antigens "early CDT lung" was approved by FDA in 2012 to detect autoantibodies directed to TAAs and assess the risk of lung cancer in the group of heavy smokers. Therefore, it is important to search for the new TAAs for creation of more sensitive antigenic panels, which will be able to discriminate cancer patients and healthy individuals. Moreover, investigation of antigens immunogenicity considering clinical and molecular characteristics of tumors including stage, histological type, receptor status, and grade expands and clarifies their use as biomarkers for cancer diagnostics.

Identification and characterization of TAAs and their autoantibodies as molecular markers of human malignancies are the long-standing goal of our Cell Signaling Department. Up to date, we have identified 81 autoantigens of colon, thyroid, and breast tumors by SEREX (serological analysis of recombinant cDNA expression libraries) approach [1017]. The preliminary phage based allogenic screening showed that 32 out of 81 SEREX-identified autoantigens had cancerrelated serological profile and reacted only with sera of cancer patients [11, 15]. Among these immunoreactive autoantigens, there are 2 proteins; in particular, Zuotin-related factor 1 (ZRF1) and R motif-containing protein 1 (KRR1) (clones KYMBC 29-88-1 and KY-MBC 18-53-1) were identified during the screening of cDNA library from medullary breast carcinoma. This study aims to confirm cancer-related serological profile of ZRF1 and KRR1 in large-scale ELISA screening of their recombinant analogues with sera of breast cancer patients of different histological types and tumor grades compared with sera of healthy individuals.

\section{Methods}

2.1. Blood Serum Samples of Patients. In total, 142 patients with newly diagnosed breast tumors including 22 patients with nonmalignant fibroadenoma (FA) and 120 patients with malignant tumors, namely, 87 patients who suffered from invasive ductal carcinoma (IDC), 23 who had invasive lobular carcinomas (ILC), and 10 who had medullary breast carcinomas (MBC), were recruited for this study (Table 1). 56 patients without neoplasm were involved as a control group.

Serum samples were taken by standard phlebotomy without the use of anticoagulants. Blood samples were kept at room temperature for 1 hour to form a fibrinogenic clot and then centrifuged for $15 \mathrm{~min}$ at $2500 \times \mathrm{g}$. Serum was pulled out, diluted with glycerol $(1: 1)$, divided into aliquots, and stored at $-20^{\circ} \mathrm{C}$.
Patient's tumors have been diagnosed using standard morphological and clinical criteria in Dnipropetrovsk City Oncology Center (Dnipropetrovs'k, Ukraine) and the National Cancer Institute, the Ministry of Health of Ukraine [18], Institute of Molecular Biology and Genetics, NASU (Kyiv, Ukraine), the Clinical Oncological Center (Dnipropetrovsk, Ukraine), and National Cancer Institute (Kyiv, Ukraine), and informed consent was obtained from all patients.

2.2. Cloning of TAAs cDNA in Bacteria and Purification of Correspondent Recombinant Proteins. cDNAs of antigens ZRF1 and KRR1 were amplified from original recombinant ZRF1/pBK-CMV and KRR1/pBK-CMV plasmids and inserted to pET-28b expression vector by standard cloning technique using Notl and Sall exonucleases. These cDNAs which were identified during SEREX screening are not fulllength but they covered the most of coding sequences (CDS) of cDNAs (Table 2). cDNAs of ZRF1 and KRR1 antigens were inserted into expression vector pET-28b in the frame with 6His-tag (Table 2) and expressed in E. coli Rosetta (DE3) pLysS cells. Affine purified recombinant proteins were used in ELISA screening with sera of breast tumors patients and healthy donors.

Expression of His-tag fused recombinant proteins was induced by $1 \mathrm{mM}$ IPTG at $37^{\circ} \mathrm{C}$ for $4 \mathrm{~h}$ in E. coli Rosetta (DE3) pLys $S$ cells transformed by correspondent recombinant plasmids. Recombinant proteins were affinity purified using NiNTA-agarose according to manufacturer protocol. Purity of proteins was analyzed by SDS-PAGE.

2.3. Enzyme-Linked Immunosorbent Assay (ELISA). Detection of autoantibodies in sera of breast cancer patients was carried out by ELISA using 96-well plate (Sarstedt, USA). Recombinant $6 \mathrm{His}$-fused proteins $(0,3 \mu \mathrm{g})$ were added to the wells in phosphate buffer (PBS), $\mathrm{pH} 7,4$ (3,2 $\mathrm{mM} \mathrm{Na}_{2} \mathrm{HPO}_{4}$; $0,5 \mathrm{mM} \mathrm{KH} \mathrm{KH}_{4} ; 1,3 \mathrm{mM} \mathrm{KCl} ; 135 \mathrm{mM} \mathrm{NaCl}$ ); sorption was carried out during the night at $+4^{\circ} \mathrm{C}$. The plates were washed with $0.1 \%$ PBS-T (phosphate buffer with $0.1 \%$ Tween), followed by blocking of binding sites with $0.1 \%$ PBS-T and $5 \%$ casein hydrolyzate (USB, USA) for $2 \mathrm{~h}$ at $37^{\circ} \mathrm{C}$. Serum samples (at a dilution of $1: 100$ in phosphate buffer with $0.5 \%$ casein hydrolyzate) were dispensed in duplicate of $100 \mu \mathrm{L}$ per well and incubated for $90 \mathrm{~min}$ at $37^{\circ} \mathrm{C}$. After four washings, antibodies conjugated to horseradish peroxidase (Jackson ImmunoResearch, USA) and directed against Fc-fragment of human IgG were added upon dilution of $1: 10^{4}$ in phosphate buffer with $1 \%$ casein hydrolyzate and incubated for $1 \mathrm{~h}$ at $37^{\circ} \mathrm{C}$. After washing, $100 \mu \mathrm{L}$ of chromogenic substrate ABTS (2,2'-azino-bis (3-ethylbenzothiazoline-6-sulphonic acid)) (Sigma, USA) was added per well and incubated for $30 \mathrm{~min}$ at $37^{\circ} \mathrm{C}$. Optical density (OD) was determined at $A_{410}$ using a spectrophotometer BioTek (USA).

2.4. Statistical Analysis. Primary data systematization and analysis were performed using Excel software (Microsoft Office, 2007). The antibody test (ELISA) was positive if optical density (OD) of serum samples exceeded the received threshold. The threshold of the test was adopted as the mean 
TABLE 1: Characteristics of tumor patients and healthy donors.

Characteristics

Samples description

(1) Group of healthy donors

Number of healthy women

56

Age at diagnosis, years (range, mean $\pm \mathrm{SD}$ )

The number of healthy donors 45 years old or older

The number of healthy donors younger than 45 years

(2) Group of patients with benign breast tumors

Number of fibroadenoma patients

Age at diagnosis, years (range, mean $\pm \mathrm{SD}$ )

The number of fibroadenoma patients 45 years old or older

The number of fibroadenoma patients younger than 45 years

(3) Group of cancer patients

Number of patients

Age at diagnosis, years (range, mean \pm SD)

The number of cancer patients 45 years old or older

The number of cancer patients younger than 45 years

Tumor type

Invasive ductal breast carcinoma

Invasive lobular breast carcinoma

Medullary breast cancer

Tumor grade (\% from general)

ER-status (\%)

Positive

Negative

PR-status (\%)

Positive

Negative

HER-2/neu status (\%)

Positive

Negative

Lymphoid nodes status (\% of positive)

SD: standard deviation.

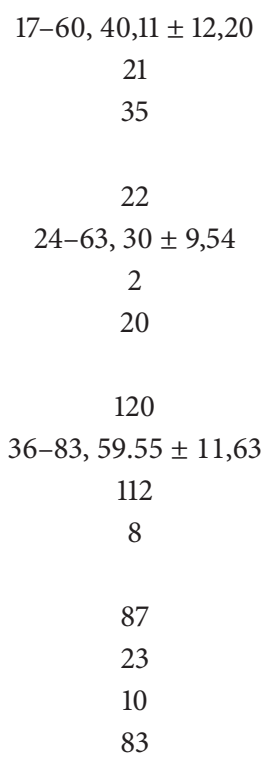

Grade 1: 4,82

Grade 2: 37,35

Grade 3: 57,83

52

71,1

28,9

52

30,8

69,2

52

30,8

69,2

28,8

TABLE 2: Antigens included in serological screening.

\begin{tabular}{|c|c|c|c|c|c|}
\hline Antigen & Full name & $\begin{array}{c}\text { cDNA reference } \\
\text { sequence }(\mathrm{NCBI} d \mathrm{~d})\end{array}$ & $\begin{array}{l}\text { cDNA-fragment according } \\
\text { to correspondent NCBI } \\
\text { reference sequence, bp }\end{array}$ & Vector & $\begin{array}{c}\text { MW of recombinant } \\
\text { protein, } \mathrm{kDa} \text { (with } \\
6 \text { His-tag) }\end{array}$ \\
\hline ZRF1 & Zuotin-related factor 1 & $\begin{array}{c}\text { NM } 014377.1 \\
\text { CDS } 252 \cdots 2117\end{array}$ & $942-2117$ & pET-28b (6His-tag) & 47,52 \\
\hline KRR1 & $\mathrm{R}$ motif-containing protein 1 & $\begin{array}{l}\text { NM } 007043.6 \\
\text { CDS } 42 \cdots 1187\end{array}$ & $42-1161$ & pET-28b (6His-tag) & 46,31 \\
\hline
\end{tabular}

plus 2 standard deviations of the OD values of all healthy donors' samples. To determine whether the frequency of autoantibodies to each of two TAAs was significantly higher in sera of breast cancer patients compared with sera of healthy donors, data were analyzed using the chi-squared test and $t$ test. In view of great age variety in group of healthy donors and groups of breast cancer and fibroadenoma patients, the comparisons were performed between age-matched groups.
For groups that include 5 or less members, statistical analysis was not executed.

2.5. Bioinformatics Analysis. Bioinformatic analysis of SEREX clones sequences was performed using bioinformatics tools provided by NCBI (http://www.ncbi.nlm.nih.gov/), GeneCards (http://www.genecards.org/), SEREX (http://www .ludwig-sun5.unil.ch/CancerImmunomeDB/), and CLUSTAL 
TABLE 3: Frequency of antibody response toward KRR1 and ZRF1 antigens in the sera of patients of different histological types of breast tumors in view of age*.

\begin{tabular}{lccccccc}
\hline Antigen & HD over 45 & HD under 45 & The number of positive sera ${ }^{* *}, n(\%)$ \\
& $(n=21)$ & $(n=35)$ & $(n=112)$ & $\begin{array}{c}\text { MBC over 45 } \\
(n=7)\end{array}$ & $\begin{array}{c}\text { IDC over 45 } \\
(n=82)\end{array}$ & $\begin{array}{c}\text { ILC over 45 } \\
(n=23)\end{array}$ & $\begin{array}{c}\text { FA under 45 } \\
(n=20)\end{array}$ \\
\hline$Z R F 1$ & $1(4,76)$ & $1(2.86)$ & $28(25,00)^{\dagger}$ & $1(14,29)$ & $22(26,83)^{\dagger}$ & $5(21,74)$ & $2(10,00)$ \\
Mean \pm SD & $0,1513 \pm 0,057$ & $0,1446 \pm 0,049$ & $0,2242 \pm 0,132$ & $0,20534 \pm 0,061$ & $0,2383 \pm 0,144$ & $0,18 \pm 0,084$ & $0,1706 \pm 0,094$ \\
KRR1 & $0(0)$ & $2(5,71)$ & $23(20,54)^{\dagger}$ & $1(14,29)$ & $21(25,61)^{\dagger}$ & $1(7,14)$ & $0(0)$ \\
Mean \pm SD & $0,2288 \pm 0,080$ & $0,2403 \pm 0,060$ & $0,3246+0,080$ & $0,2843 \pm 0,072$ & $0,3430 \pm 0,077$ & $0,2712 \pm 0,068$ & $0,1884 \pm 0,054$ \\
\hline
\end{tabular}

Notes: ${ }^{*}$ statistical analysis of frequency of antibody response was calculated between BC patients and healthy individuals cohorts over 45 years old and between FA and healthy individuals cohorts under 45 years old; ${ }^{* *}$ threshold: the mean plus $2 \mathrm{SD}$ in the group of healthy donors; ${ }^{\dagger}$ Statistically significant difference compared to healthy donors' sera $(P<0.05)$. HD: healthy donors; BC: breast cancer (IDC + ILC + MBC); MBC: medullary breast carcinoma; IDC: invasive ductal carcinoma; ILC: invasive lobular carcinoma; FA: fibroadenoma.

W (1.83) (http://embnet.vital-it.ch/software/ClustalW.html) resources.

\section{Results}

3.1. Cloning of cDNAs of KRR1 and ZRF1 Antigens, Expression, and Purification of Correspondent Recombinant Proteins. In this investigation, our attention was focused on two potential tumor-associated antigens, namely, Zuotin-related factor 1 (ZRF1) and R motif-containing protein 1 (KRR1), previously identified by SEREX analysis of medullary breast carcinoma cDNA library $[12,15]$. These antigens had tumor-associated serological profile of autoantibodies according to the data of preliminary phage based allogeneic screening with sera of medullary breast carcinoma patients [12]. Recombinant plasmids ZRF1/pBK-CMV and KRR1/pBK-CMV were obtained by "in vivo excision" procedure of cDNA of phage $\lambda$ positive clones of MBC cDNA library during previous investigation $[12,15]$.

\subsection{Analysis of Antibody Response to KRR1 and ZRF1 Recom-} binant Proteins in the Sera of Breast Tumors Patients and Healthy Donors. Recombinant proteins were analyzed in the allogenic screening using sera of patients with different histological types and tumor grades compared with sera of healthy individuals. Since the most of breast patients were over 45 years of age while the most of the fibroadenoma patients and healthy individuals were under 45 years of age, we performed comparative analysis of serum reactivity in corresponding age-matched groups. Table 3 presents the results of large-scale allogeneic screening of two investigated antigens. Increased frequency of autoantibody response to ZRF1 and KRR1 antigens was detected in $25.00 \%$ and $20.54 \%$ of breast cancer patients' sera, respectively (Table 3 ).

Analysis of antibody response frequency toward KRR1 and ZRF antigens in the sera of breast cancer patients taking into account histological type of tumors showed a statistically significant antibody response only in sera of patients with invasive ductal carcinomas. For ZRF1, significant reactivity also was shown in sera of patients with G1 and G2 tumors (Table 4).

We found no significant difference of frequency of antibody response in sera of breast cancer patients taking
TABLE 4: Frequency of antibody response toward KRR1 and ZRF1 antigens in the sera of breast cancer patients of different tumor grade.

\begin{tabular}{lcc}
\hline $\begin{array}{l}\text { Tumor grade }(n \text { : the } \\
\text { number of positive sera }\end{array}$ & \multicolumn{2}{c}{ Antigens } \\
\hline $\mathrm{G} 1+\mathrm{G} 2(n=35)$ & $42,86(15)^{\dagger}$ & KRR1 \\
$\mathrm{G} 3(n=48)$ & $18,75(9)$ & $18,75(9)$ \\
\hline
\end{tabular}

G1: grade 1 tumor, G2: grade 2 tumor, and G3: grade 3 tumor; $n$ : the number of positive sera; ${ }^{*}$ threshold: the mean plus $2 \mathrm{SD}$ in the group of healthy donors. ${ }^{\dagger}$ Statistically significant difference compared to G3 tumor grade $(P<0.05)$.

into account tumor's receptor and lymphoid nodes status.

As it was mentioned above, ZRF1 (also known as DNAJC2, MPP11) was described as SEREX-antigen in different malignancies [19-22]. Totally, 4 SEREX clones NY-SCLC6, NY-BR-13, NGO-St-58 5', and UL-CML-1 have been identified by screening of cDNA libraries from lung, breast, and stomach cancers and leukemia correspondingly (Table 5). Analysis of antibody response in sera of cancer patients toward the protein products of these clones according to literature data and our own results showed contradictory outcomes (Table 5). These data prompted us to perform analysis of available sequence of SEREX clones which encode ZRF1 (DNAJC2, MPP11) antigen.

3.3. Analysis of Sequences of SEREX Clones Identified from Different Tumors, Which Encode ZRF1 Antigen. Today there are three available cDNA sequences of ZRF1 SEREX clones: NGO-St-58, NY-BR-13, and KY-MBC 29-88-1. The NY-BR-13 clone was identified by Matthew Scanlan (Ludwig Institute for Cancer Research, NY, USA) by screening of breast cancer cDNA library and NGO-St-58 $5^{\prime}$ clone was identified by Yuichi Obata (Aichi Cancer Center, Tokyo, Japan) by screening of stomach cancer cDNA library (Table 5).

Comparative analysis of sequences of these clones revealed $159 \mathrm{bp}$ length insertions in the sequences of clones NGO-St-58 and KY-MBC 29-88-1 that was absent in the sequence of NY-BR-13 clone (Figure 1). BLAST analysis of cDNA sequences of NY-BR-13, NGO-St-58, and KY-MBC 
TABLE 5: SEREX clones which encode ZRF1 (DNAJC2, MPP11) antigen.

\begin{tabular}{|c|c|c|c|}
\hline Clone name & Source & $\begin{array}{l}\text { Frequency of antibody response in } \\
\text { cancer patients sera versus healthy } \\
\text { donors sera }\end{array}$ & Publication \\
\hline NY-SCLC-6 & $\begin{array}{l}\text { NCI-H740 and } \\
\text { SK-LC-13 cell } \\
\text { lines }\end{array}$ & N/A & Güre et al., PNAS, 2000; 97: 4198-4203 [19] \\
\hline NY-BR-13 & Breast cancer & $3 / 10 \mathrm{BC}$ versus $3 / 12 \mathrm{HD}$ & $\begin{array}{l}\text { Scanlan et al., Cancer Immun 2001: 4-21 [20]. } \\
\text { (Ludwig-sun5.unil.ch/CancerImmunomeDB/) }\end{array}$ \\
\hline NGO-St-58, 5 & Stomach cancer & $4 / 13 \mathrm{SC}$ versus $3 / 16 \mathrm{HD}$ & $\begin{array}{l}\text { Obata et al. (Ludwig-sun5.unil.ch/CancerImmunomeDB/) } \\
\text { [21] }\end{array}$ \\
\hline UL-CML-1 & $\begin{array}{l}\text { K562 cell line } \\
\text { from CML } \\
\text { patient }\end{array}$ & $\begin{array}{l}\text { 7/19 AML, 6/16 CML, 4/10 RCC, } 3 / 6 \\
\mathrm{MM}, 3 / 12 \mathrm{BC}, 3 / 10 \text { OC versus 0/20 HD }\end{array}$ & Greiner et al., Int. J. Cancer 2003; 106: 224-231 [22]. \\
\hline $\begin{array}{l}\text { KY-MBC } \\
29-88-1\end{array}$ & Breast cancer & $\begin{array}{l}\text { 33/120 BC (including 1/10 MBC, 27/87 } \\
\text { IDC, 5/23 ILC), 3/22 FA versus 2/50 HD }\end{array}$ & Present paper \\
\hline
\end{tabular}

BC: breast cancer patients; HD: healthy donors; SC: stomach cancer; RCC: renal cell carcinoma; MM: metastatic melanoma; AML: acute myeloid leukemia; CML: chronic myeloid leukemia; OC: ovarian carcinoma.

\begin{tabular}{|c|c|}
\hline $\begin{array}{l}\text { KY-MBC-29-88-1 } \\
\text { NGO-ST-58 } \\
\text { NY-BR-13 }\end{array}$ & 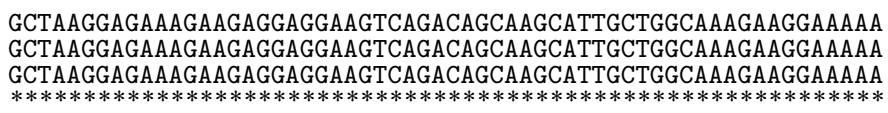 \\
\hline $\begin{array}{l}\text { KY-MBC-29-88-1 } \\
\text { NGO-ST-58 } \\
\text { NY-BR-13 }\end{array}$ & 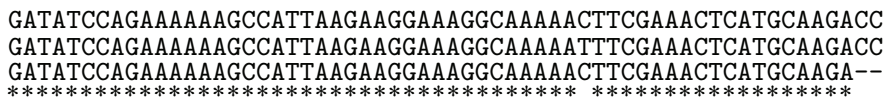 \\
\hline $\begin{array}{l}\text { KY-MBC-29-88-1 } \\
\text { NGO-ST-58 } \\
\text { NY-BR-13 }\end{array}$ & $\begin{array}{l}\text { TGGAATCATTTTTCTGATAATGAGGCAGAGCGGGTTAAAATGATGGAAGAAGTGGAAAAA } \\
\text { TGGAATCATTTTTCTGATAATGAGGCAGAGCGGGTTAAAATGATGGAAGAAGTGGAAAAA }\end{array}$ \\
\hline $\begin{array}{l}\text { KY-MBC-29-88-1 } \\
\text { NGO-ST-58 } \\
\text { NY-BR-13 }\end{array}$ & $\begin{array}{l}\text { CTTTGTGATCGGCTTGAACTGGCAAGCTTACAGTGCTTGAATGAAACACTCACATCATGC } \\
\text { CTTTGTGATCGGCTTGAACTGGCAAGCTTACAGTGCTTGAATGAAACACTCACATCATGC }\end{array}$ \\
\hline $\begin{array}{l}\text { KY-MBC-29-88-1 } \\
\text { NGO-ST-58 } \\
\text { NY-BR-13 }\end{array}$ & $\begin{array}{l}\text { ACAAAAGAAGTAGGAAAGGCTGCTTTGGAAAAACAGATAGAAGAAATAAATGAGCAAATC } \\
\text { ACAAAAGAAGTNGGAAAGGTGCTTTGGAAAACAGATAGAAGAAATAAATGAGCAAATC } \\
- \\
* * * * * * * * * * * * * * * * * * * *\end{array}$ \\
\hline $\begin{array}{l}\text { KY-MBC-29-88-1 } \\
\text { NGO-ST-58 } \\
\text { NY-BR-13 }\end{array}$ & $\begin{array}{l}\text { AGAAAAGAGAAAGAGGAAGCTGAGGCTCGTATGCGACAAGCATCTAAGAACACAGAGAAA } \\
\text { AGAAAAGAGAAAGAGGAAGCTGAGGCTCGTATGCGACAAGCATCTAAGAACACAGAGAAA } \\
\text { AGAAAAGAGAAAGAGGAAGCTGAGGCTCGTATGCGACAAGCATCTAAGAACACAGAGAAA }\end{array}$ \\
\hline
\end{tabular}

FIGURE 1: Part of multiple alignment of KY-MBC 29-88-1, NGO-St-58, and NY-BR-13 clones sequences by CLUSTAL W (1.83) analysis.

29-88-1 clones showed that sequences of clones NGO-St58 and KY-MBC 29-88-1 correspond to the longer mRNA transcript 1 of DNAJC2 gene (Ref. sec. NM_014377.1), while full-length sequence of NY-BR-13 clone (reference sequence X98260) corresponds to the shorter mRNA transcript 2 of DNAJC2 gene (Ref. sec. NM_001129887.1) that differs by $159 \mathrm{bp}$ (Table 6). Unfortunately, we could not analyze sequences of UL-CML-1 and NY-SCLC- 6 clones because they were not found $[19,22]$.

Obtained data indicate that at least three SEREX clones KY-MBC 29-88-1, NGO-St-58, and NY-BR-13 correspond to different mRNA transcripts of DNAJC2 gene, which encode in turn two different protein isoforms (Table 6).

\section{Discussion}

Tumor-associated antigens and their correspondent autoantibodies are promising molecular markers for diagnostics and therapy of human malignancy. Today more than 2000 TAAs have been identified by different approaches including SEREX, SEPRA, phage display, microarray, and other high throughput technologies $[14,23]$. Previously, we have described 81 autoantigens identified from breast, colon, and thyroid cancers by SEREX approach $[10,12,13,15,16]$. Original modification of SEREX technique for the generation of medullary breast carcinoma cDNA expression library depleted from IgG genes has been developed [12]. As a result, MBC cDNA library suitable for serological screening was created and $41 \mathrm{MBC}$-derived autoantigens have been identified [12]. Preliminary phage based allogenic screening revealed that 18 of them had exhibited cancer-related autoantibody profile and reacted only with sera of medullary breast carcinoma patients but not with sera of healthy donors $[12,15]$. Here, we studied immunogenicity of 2 out of 18 $\mathrm{MBC}$ antigens, namely, ZRF1 and KRR1, in sera of patients 


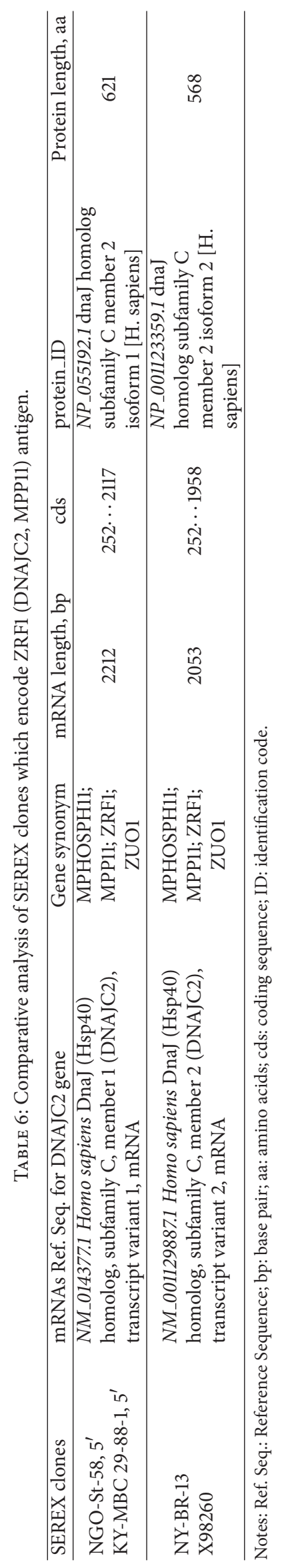


with breast tumors of different histological types and grades compared with sera of age-matched healthy donors using large-scale allogeneic screening performed by ELISA.

Zuotin-related factor 1 (ZRF1) and R motif-containing protein 1 (KRR1) have a multifunctional role in different cell processes including malignant transformation. KRR1 is a factor involved in a process of ribosomal assembly and essential for cell viability [24]. Some data demonstrate that KRR1 is highly expressed in dividing cells; moreover, its expression ceases almost completely, when cells enter the stationary phase. In vivo depletion of KRR1 leads to vigorous reduction of $40 \mathrm{~S}$ ribosomal subunits due to defective $18 \mathrm{~S}$ rRNA synthesis [24]. There is a little data about KRR1 function in tumors. It was suggested only that KRR1 is associated with metastasis in malignant fibrous histiocytoma [23]. During this study, we identified for the first time increased frequency of antibody response toward KRR1 antigen in sera of breast cancer patients and in sera of patients with invasive ductal breast carcinoma compared with sera of healthy women.

Zuotin-related factor 1 (ZRF1), that has several names including DNAJC2 (Hsp40); ZUO1; MPP11; MPHOSPH11 and is encoded by DNAJC2 gene, is a member of the Mphase phosphoprotein (MPP) family, which acts as both a chaperone in the cytosol [25] and a chromatin regulator in the nucleus [26]. The chromosomal aberrations involving this gene are associated with primary head and neck squamous cell carcinomas (HNSCC) [27]. A tumor-specific high level of MPP11 expression in HNSCC was shown [27]. Cluster analysis showed that gene, which is EST, is moderately similar to ZFR1, displayed differential expression in lymph node positive and negative gastric tumors [28]. The mutation in the human Mpp11 J protein (ZRF1) which leads to loss of function of this protein has been described [25]. Some authors concluded that ZRF1 involved in regulation of cellular proliferation and senescence and alterations in ZRF1 may contribute to tumorigenesis [29]. Analysis performed during this study showed increased frequency of antibody response toward ZRF1 antigen in sera of patients with invasive ductal carcinomas. Greiner et al. [22] also demonstrated significant antibody response toward MPP11 in the sera of breast cancer patients but not in sera of healthy volunteers. These data contradict the data of Obata et al. [21] and Scanlan et al. [20] who both failed to show increased immunogenicity of ZRF1 antigen in sera of stomach and breast cancer patients correspondingly compared with sera of healthy women. This concordance was explained by Greiner in part by different cDNAs lengths of SEREX clones that might result in a various conformation of the gene's protein products and subsequently in a different epitope presentation to IgG antibodies [22].

We performed analysis of SEREX clones (NY-BR-13, NGO-St-58, and KY-MBC 29-88-1) sequences coding ZRF1 and revealed $159 \mathrm{bp}$ lengths insert in the sequences of clones NGO-St-58 and KY-MBC 29-88-1. BLAST analysis indicated that sequences of clones NGO-St-58 and KY-MBC 29-88-1 correspond to longer transcript variant 1 of DNAJC2 gene, while sequence of NY-BR-13 clone corresponds to shorter transcript variant 2 of DNAJC2 gene. Thus, these data allowed us to suppose that NGO-St-58, KY-MBC 29-88-1, and NYBR-13 SEREX clones correspond to 2 alternatively spliced
mRNA isoforms of DNAJC2 gene, that encode two different protein isoforms. This observation, in part, can explain differences in allogenic screening results of these SEREX clones performed by different researchers. Due to previous literature data and data obtained in this study, we suggest that different protein isoforms and/or mutant forms of DNAJC2 could be differently expressed in various tumors and may differ in immunogenicity. In addition, increased frequency of antibody response in sera of patients with less aggressive tumors (G1 and G2) shown during this study allowed us to suppose that autoantibodies toward ZFR1 antigen may appear at the initial steps of breast cancer malignancy and represent potential markers for early cancer diagnostics.

So, analysis of antibody response in sera of breast cancer patients with tumors of different clinical and molecular characteristics showed that KRR1 and ZRF1 antigens and autoantibodies thereto are potential breast tumor markers, which could be important for creation of new antigenic/autoantibody signatures for breast tumor detection including early breast cancer diagnostics and/or for improving already existing ones. Comparative analysis of ZRF1 SEREX clones showed that they code different protein isoforms of DNAJC2 gene in different tumors and cell lines.

\section{Competing Interests}

The authors declare that there is no conflict of interests regarding the publication of this paper.

\section{Acknowledgments}

This work was supported by Russian Science Foundation (Project 15-15-20032).

\section{References}

[1] A. Bleyer and H. G. Welch, "Effect of three decades of screening mammography on breast-cancer incidence," The New England Journal of Medicine, vol. 367, no. 21, pp. 1998-2005, 2012.

[2] B. Weigelt, F. C. Geyer, and J. S. Reis-Filho, "Histological types of breast cancer: how special are they?" Molecular Oncology, vol. 4, no. 3, pp. 192-208, 2010.

[3] N. T. Brewer, T. Salz, and S. E. Lillie, "Systematic review: the long-term effects of false-positive mammograms," Annals of Internal Medicine, vol. 146, no. 7, pp. 502-510, 2007.

[4] A. Coolen, K. Leunen, J. Menten, W. van Steenbergen, and P. Neven, "False-negative tests in breast cancer management," Netherlands Journal of Medicine, vol. 69, no. 7, pp. 324-329, 2011.

[5] C. Desmetz, A. Mange, T. Maudelonde, and J. Solassol, "Autoantibody signatures: progress and perspectives for early cancer detection," Journal of Cellular and Molecular Medicine, vol. 15, no. 10, pp. 2013-2024, 2011.

[6] H. Lu and V. Goodell, "Humoral immunity directed against tumor-associated antigens as potential biomarkers for the early diagnosis of cancer," Journal of Proteome Research, vol. 45, pp. 88-94, 2008.

[7] E. Piura and B. Piura, "Autoantibodies to tailor-made panels of tumor-associated antigens in breast carcinoma," Journal of Oncology, vol. 2011, Article ID 982425, 7 pages, 2011. 
[8] K. Looi, R. Megliorino, F.-D. Shi, X.-X. Peng, Y. Chen, and J.-Y. Zhang, "Humoral immune response to p16, a cyclin-dependent kinase inhibitor in human malignancies," Oncology Reports, vol. 16, no. 5, pp. 1105-1110, 2006.

[9] A. Yagihashi, T. Ohmura, K. Asanuma et al., "Detection of autoantibodies to survivin and livin in sera from patients with breast cancer," Clinica Chimica Acta, vol. 362, no. 1-2, pp. 125130, 2005.

[10] O. Garifulin, V. Kykot, N. Gridina et al., "Application of SEREXanalysis on colon cancer cases," Experimental Oncology, vol. 2, pp. 128-131, 2003.

[11] R. Kiyamova, O. Garifulin, V. Gryshkova et al., "Preliminary study of thyroid and colon cancers-associated antigens and their cognate autoantibodies as potential cancer biomarkers," Biomarkers, vol. 17, no. 4, pp. 362-371, 2012.

[12] R. Kiyamova, O. Kostianets, S. Malyuchik et al., "Identification of tumor-associated antigens from medullary breast carcinoma by a modified SEREX approach," Molecular Biotechnology, vol. 46, no. 2, pp. 105-112, 2010.

[13] R. G. Kyyamova and V. V. Filonenko, "Tumor-associated antigens and development of immunotherapeutics strategies," Biopolymers and Cell, vol. 21, no. 3, pp. 220-229, 2005.

[14] C. S. Klade, "Proteomics approaches towards antigen discovery and vaccine development," Current Opinion in Molecular Therapeutics, vol. 4, no. 3, pp. 216-223, 2002.

[15] O. Kostianets, M. Shyian, D. Sergiy et al., "Serological analysis of SEREX-defined medullary breast carcinoma-associated antigens," Cancer Investigation, vol. 30, no. 7, pp. 519-527, 2012.

[16] N. V. Rodnin, I. O. Tykhonkova, R. G. Kyyamova, O. M. Garifulin, I. T. Gout, and V. V. Filonenko, "Identification of tumorassociated antigens in human thyroid papillar carcinoma," Biopolymers \& Cell, vol. 19, no. 6, pp. 541-547, 2003.

[17] M. A. Shyian, O. I. Kostianets, O. Y. Tsuvariev et al., "The peculiarities of gene expression of medullary breast carcinoma tumor-associated antigens in different types of breast tumors," Biopolymers and Cell, vol. 28, no. 5, pp. 381-388, 2012.

[18] F. Tavassoli and P. Devilee, World Health Organization Classification of Tumours Pathology and Genetics of Tumours of the Breast and Female Genital Organs, vol. 5, IARC Press, Lyon, France, 2003.

[19] A. O. Güre, E. Stockert, M. J. Scanlan et al., "Serological identification of embryonic neural proteins as highly immunogenic tumor antigens in small cell lung cancer," Proceedings of the National Academy of Sciences of the United States of America, vol. 97, no. 8, pp. 4198-4203, 2000.

[20] M. J. Scanlan, I. Gout, C. M. Gordon et al., "Humoral immunity to human breast cancer: antigen definition and quantitative analysis of mRNA expression," Cancer Immunity, vol. 1, pp. 4$21,2001$.

[21] Y. Obata, T. Takahashi, J. Sakamoto et al., "Serex analysis of gastric cancer antigens," Cancer Chemotherapy and Pharmacology, vol. 46, supplement 1, pp. S37-S42, 2000.

[22] J. Greiner, M. Ringhoffer, M. Taniguchi et al., "Characterization of several leukemia-associated antigens inducing humoral immune responses in acute and chronic myeloid leukemia," International Journal of Cancer, vol. 106, no. 2, pp. 224-231, 2003.

[23] D. Adrien, K.-H. Ludger, S. Ingo et al., "Malignant fibrous histiocytoma-pleomorphic sarcoma, NOS gene expression, histology, and clinical course. A Pilot Study," Langenbeck's Archives of Surgery, vol. 395, no. 3, pp. 261-275, 2010.
[24] R. Gromadka and J. Rytka, "The KRR1 gene encodes a protein required for $18 \mathrm{~S}$ rRNA synthesis and $40 \mathrm{~S}$ ribosomal subunit assembly in Saccharomyces cerevisiae," Acta Biochimica Polonica, vol. 47, no. 4, pp. 993-1005, 2000.

[25] H. A. Hundley, W. Walter, S. Bairstow, and E. A. Craig, "Human Mpp11 J protein: ribosome-tethered molecular chaperons are ubiquitous," Science, vol. 308, no. 5724, pp. 1032-1034, 2005.

[26] H. Richly, L. Rocha-Viegas, J. D. Ribeiro et al., "Transcriptional activation of polycomb-repressed genes by ZRF1," Nature, vol. 468, pp. 1124-1128, 2010.

[27] V. A. Resto, O. L. Caballero, M. R. Buta et al., "A putative oncogenic role for MPP11 in head and neck squamous cell cancer," Cancer Research, vol. 60, no. 19, pp. 5529-5535, 2000.

[28] A. Marchet, S. Mocellin, C. Belluco et al., "Gene expression profile of primary gastric cancer: towards the prediction of lymph node status," Annals of Surgical Oncology, vol. 14, no. 3, pp. 10581064, 2007.

[29] S. Demajo, I. Uribesalgo, A. Gutiérrez et al., "ZRF1 controls the retinoic acid pathway and regulates leukemogenic potential in acute myeloid leukemia," Oncogene, vol. 33, pp. 5501-5510, 2014. 


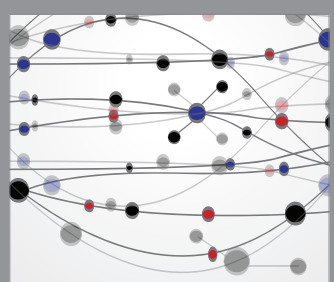

The Scientific World Journal
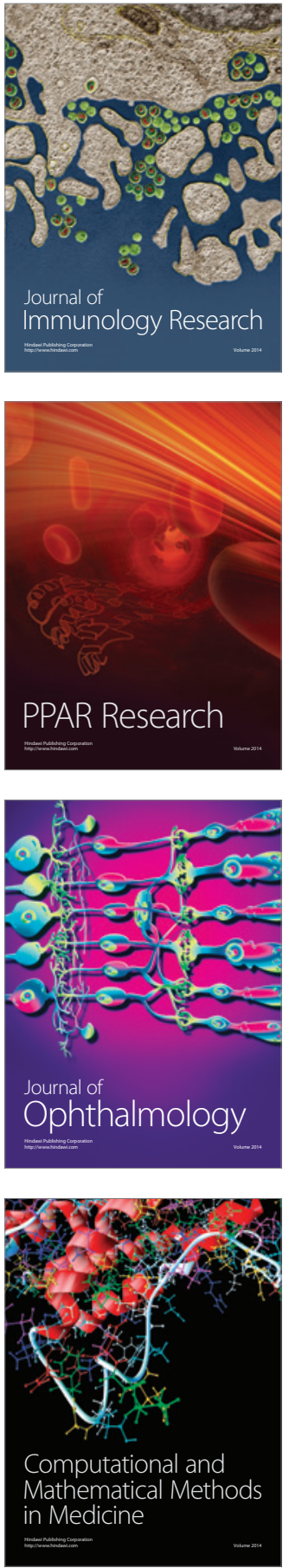

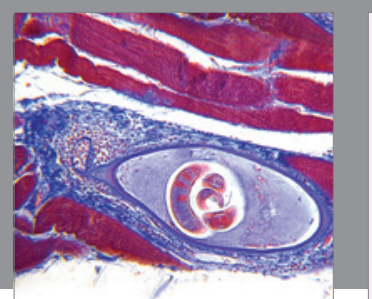

Gastroenterology Research and Practice

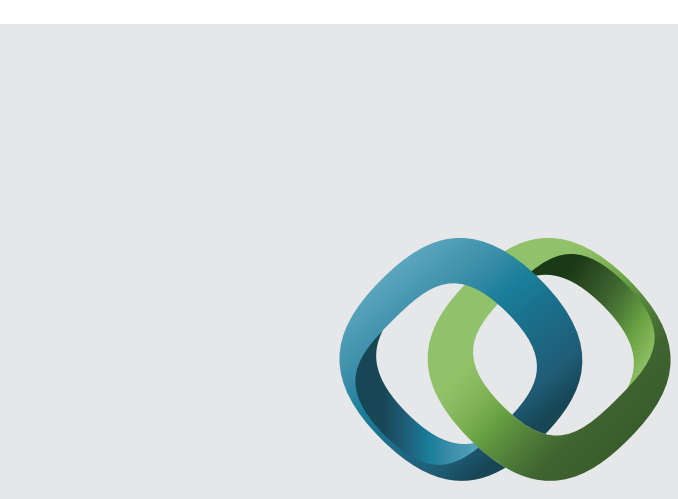

\section{Hindawi}

Submit your manuscripts at

http://www.hindawi.com
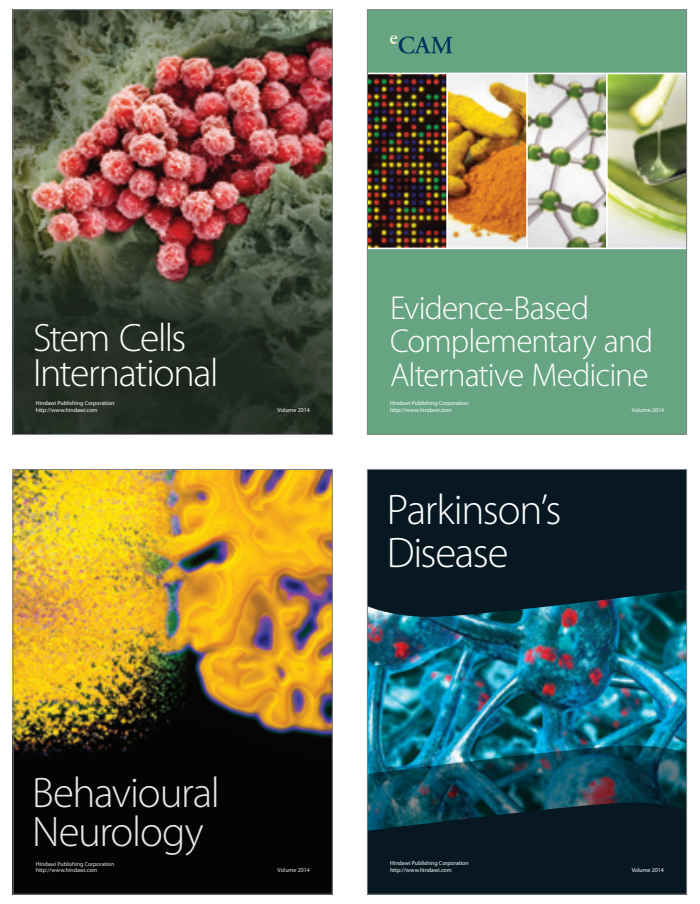
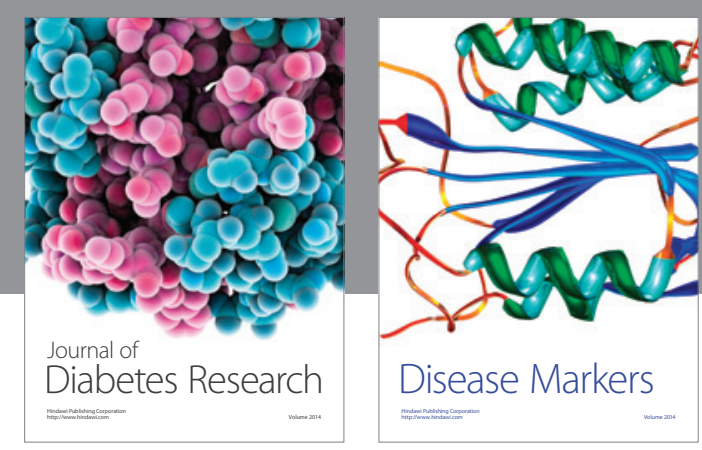

Disease Markers
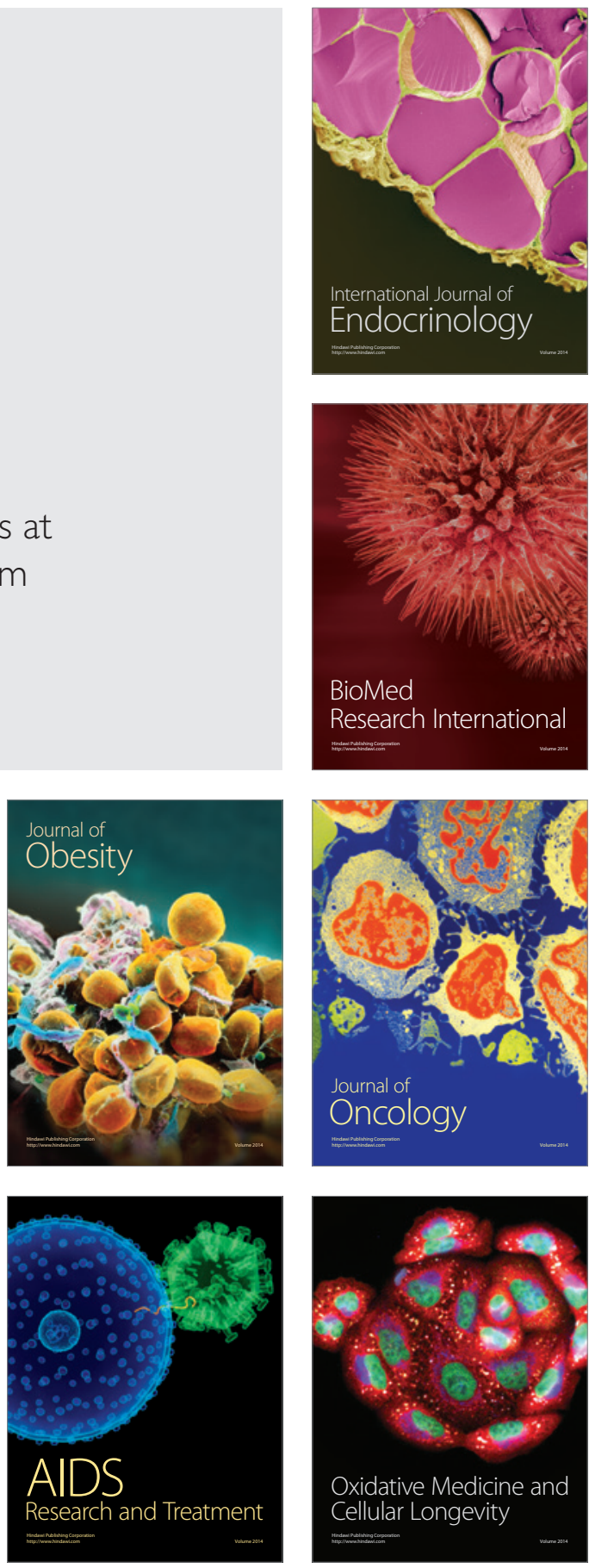